\title{
„Zu den typischen in der höchstrichterlichen Rechtsprechung behandelten Folgen der Beendigung einer Freiberuflersozietät“"
}

\author{
Prof. Dr. Wulf GoetTe, Ettlingen*
}

Inhaltsübersicht

ZGR 2017, 426-437

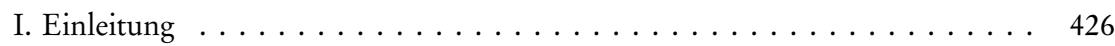

II. Grundlagen . . . . . . . . . . . . . . . . . . . . . . . . 428

1. Gründe und Form der Beendigung . . . . . . . . . . . . . . . . . . . . . . . . 428

2. Auflösung der Gesellschaft . . . . . . . . . . . . . . . . . . . . . . . . . . 430

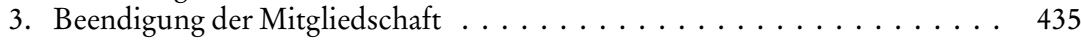

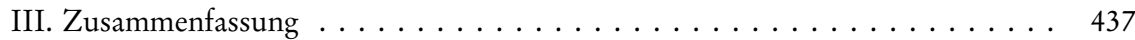

Freiberufler üben, wenn sie sich zur gemeinsamen Berufsausübung entschließen, ibre Tätigkeit immer noch weit überwiegend in personengesellschaftsrechtlicher Form aus. Hier steht die BGBGesellschaft im Vordergrund. Wegen oftmals unzureichender vertraglicher Vorsorge wirft die Beendigung solcher Freiberuflersozietäten nicht selten schwierige Fragen auf, die die höchstrichterliche Rechtsprechung immer wieder beschäftigt haben. Einige dieser Probleme und ihrer Lösung versucht der nachfolgende Beitrag darzustellen.

\section{Einleitung}

Früher war die BGB-Gesellschaft für Freiberufler die typische Berufsausübungsgemeinschaft, sie hat auch in Zeiten der Verwendung von Aktiengesellschaften, Gesellschaften mit beschränkter Haftung oder europäischen Rechtsformen wie der llp - zumindest für die kleinen und mittelgroßen Einheiten für diese Berufsgruppe wenig an Attraktivität verloren. Zu der personalistisch geprägten, besonders auf gegenseitiges Vertrauen gestützten, aktive Mitarbeit voraussetzenden und prinzipiell auf Dauer angelegten Zusammenarbeit passt die personengesellschaftsrechtliche Struktur besser. Ohne zu viele Fesseln des zwingenden Rechts lassen sich individuell zugeschnittene, privatautonom gestaltete Regeln schaffen, die für die notwendige Flexibilität sorgen. Das gilt in ähnlicher Weise wie für die BGB-Gesellschaft auch für die inzwischen weit

* Prof. Dr. Wulf GoetTe war bis 2010 Vorsitzender Richter des II. Zivilsenats des Bundesgerichtshofs und ist Honorarprofessor der Universität Heidelberg. 
verbreitete Partnerschaftsgesellschaft, deren Variante als Gesellschaft mit beschränkter Berufshaftung zusätzlich die mit der gemeinsamen Berufstätigkeit heraufbeschworenen Gefahren mindert. Wegen dieser zu einer Freiberuflergesellschaft besser passenden Struktur hat die BGB-Gesellschaft nach wie vor große Anziehungskraft.

Die personalistische Struktur und die auf Dauer angelegte Zusammenarbeit bringen indessen nicht nur Vorzüge mit sich, das in ihnen zum Ausdruck kommende beharrende Element kann von den einzelnen Mitgliedern auch als hemmend empfunden werden. Das gilt vor allem, wenn bei einzelnen Mitgliedern der Wunsch nach größtmöglicher Flexibilität und Bindungsfreiheit besteht. Probleme können auch dadurch entstehen, dass sich die Lebensentwürfe der Partner auseinander entwickeln, dass der unter den Gesellschaftern zu verteilende Kuchen kleiner wird oder die Gewinnverteilungsquoten nicht mehr dem Maß der eigenen Beiträge zum Ganzen entsprechen und der anfangs bestehende Kitt der „persönlichen Chemie“ im Laufe der Zeit so bröckelig geworden ist, dass notwendige Anpassungen konsensual nicht mehr herbeigeführt werden können. Nicht nur hieraus können Unzuträglichkeiten entstehen, sondern - jenseits des Materiellen - etwa auch aus einem Generationenkonflikt zwischen älteren und jüngeren Sozien, z.B. weil der Senior auch Jahre nach dem Eintritt des jüngeren Partners das „Sagen“ behalten will, bestimmte Mandate für sich beansprucht, aber Entwicklungsmöglichkeiten der Jüngeren nicht unterstützt oder sogar eifersüchtig auf deren Ansehen und beruflichen Erfolg blickt. Unter anderem dann kann es notwendig werden, die berufliche Zusammenarbeit zu beenden, woraus sich eine Reihe von - zum Teil nicht einfach zu lösenden - Problemen ergeben können; ähnliche Fragen können aber auch auftreten, wenn eine Freiberuflersozietät aus anderem Anlass beendet wird.

Der nachfolgende Überblick ist Peter Hommelhoff, dem Heidelberger Freund und langjährigen geschäftsführenden Herausgeber dieser und Ihrer Tochterzeitschrift, der ECFR, herzlich gewidmet. Die Hoffnung, dass er diesen Beitrag mit Freude lesen wird, auch wenn nicht die BGB-Gesellschaft, sondern die GmbH den Schwerpunkt seines Forschens und Lehrens bildet, ist deswegen berechtigt, weil nicht nur die $\mathrm{GmbH}$ in ihrer typischen personalistischen Struktur der BGB-Gesellschaft nahe steht und manche hier erörterten Fragen auch bei der $\mathrm{GmbH}$ auftreten, sondern vor allem weil der Jubilar über Jahrzehnte eine in der Rechtsform einer - idealistischen Zwecken dienenden BGB-Gesellschaft verfasste Herausgebergemeinschaft mit so großem Erfolg geführt hat, dass Rechtsfragen der Zusammenarbeit keine Rolle gespielt haben, aber man in der Rückschau fragen könnte: Was wäre, wenn? 


\section{Grundlagen}

\section{Gründe und Form der Beendigung}

Die Gründe für die Beendigung eines Gesellschaftsverhältnisses zwischen Freiberuflern sind ganz unterschiedlicher Art. Sie vollziehen sich auf unterschiedliche Weise und sie können auch ganz andere Rechtsfolgen nach sich ziehen.

\section{a) Befristung}

Eine auf bestimmte Zeit eingegangene Gesellschaft endet - die besonderen in $\$ \mathbb{S} 723,724$ BGB niedergelegten Kündigungsmöglichkeiten außer Betracht gelassen - jedenfalls mit Ablauf der Zeit von selbst, ohne dass es einer besonderen Handlung der Gesellschafter bedarf. Anders ist dies, wenn das Gesellschaftsverhältnis nach Ablauf der vereinbarten Zeit fortgesetzt werden soll. Dann ist zwar nicht unbedingt ein förmlicher Fortsetzungsbeschluss erforderlich ( $\$ 724$ Satz 2 BGB), die Gesellschafter müssen aber sehr wohl auch nach außen hin in irgendeiner Weise deutlich machen, dass sie die frühere Entscheidung revidieren. ${ }^{1}$

\section{b) Altersgrenze}

Ist vereinbart, dass ein Gesellschafter bei Erreichen eines bestimmten Alters ausscheiden muss, handelt es sich um einen Unterfall einer zeitlichen Befristung. Sie führt aber im Regelfall nicht zur Auflösung der Gesellschaft, sondern nur zur Beendigung der Mitgliedschaft und bringt wegen der dann fälligen Abfindungszahlung, etwaiger Versorgungsansprüche und des Eingreifens von Wettbewerbs- und Mandantenschutzklauseln mancherlei Abwicklungsprobleme mit sich.

\section{c) Tod}

Mit dem Tod eines Gesellschafters endet nicht nur selbstverständlich sein Mitgliedschaftsverhältnis, die Gesellschaft tritt auch ins Auflösungsstadium ein,

1 BGH, Urt. v. 19.6.1995 - II ZR 255/93, NJW 1995, 2843 f [Steuerberatersozietät]; C. SCHäfer, Münchener Komm. z. BGB, 7. Aufl., 2017, Vor $\$ 723$ Rdn. 11, auch zum erforderlichen Stimmenquorum und der Notwendigkeit der Abfindung eines dissentierenden Gesellschafters bei Vorhandensein einer Mehrheitsklausel. 
sofern nicht eine Fortsetzungs- oder Übernahmeklausel besteht ( $\$ 727$ BGB). In beiden Fällen haben sich die anderen Gesellschafter mit einem außenstehenden Dritten, dem Erben auseinander zu setzen.

\section{d) Insolvenz}

Nach dem Gesetz ( $\ 728$ BGB) ziehen auch die Eröffnung des Insolvenzverfahrens über das Vermögen der Gesellschaft oder eines Gesellschafters automatisch die Auflösung der Gesellschaft nach sich. Für den Fall der Gesellschafterinsolvenz können aber - und dies geschieht auch regelmäßig - schon im Voraus abweichende Regeln getroffen werden, nach denen in diesem Fall der Gesellschafter aus wichtigem Grund ausscheidet.

\section{e) Zweckerreichung und Unmöglichkeit der Zweckerreichung}

Auflösungsgrund ist ferner die Erreichung des vereinbarten Zwecks der Gesellschaft ( $\ 726 \mathrm{BGB})$, eine für eine Freiberuflersozietät wohl kaum auftretende Konstellation; anders - etwa beim Eingreifen von die Berufsausübung regelnden Umständen - kann es aber mit der Variante liegen, dass die Erreichung des Zwecks unmöglich wird.

\section{f) Ausschließungs- und Austrittskündigung}

Die praktisch häufigsten Fälle der Beendigung eines Gesellschaftsverhältnisses lassen sich unter den Oberbegriff der Kündigung zusammenfassen. Sie begegnen als Kündigung des Gesellschaftsverhältnisses, wie sie dem gesetzlichen Modell in den $\mathbb{S} 723,724$ und 725 als Regelfall entspricht, und der Kündigung des Mitgliedschaftsverhältnisses in Gestalt einer aus wichtigem Grund stattfindenden Ausschließungs- oder einer Austrittskündigung, die jeweils nur das Gesellschaftsverhältnis des Betroffenen beendet, während die Gesellschaft im Übrigen fortgesetzt wird. In den Rechtsfolgen ähnlich gestaltet sind die Fälle, in denen vereinbarungsgemäß die gesetzlich vorgesehene Auflösung der Gesellschaft ersetzt wird durch eine Regelung, nach der die Gesellschaft unter den verbleibenden Partnern fortgesetzt und der ausgeschiedene Gesellschafter, seine Erben oder seine Gläubiger abgefunden werden.

\section{g) Anschluss an Austrittskündigung}

Auch wenn ein Gesellschafter mit einer Austrittskündigung - etwa, weil er damit für ihn vorteilhafte Abfindungsregeln auslösen möchte - die Gesell- 
schaft nicht aufgelöst wissen will, kann er sich mit diesem Wunsch nicht in jedem Fall durchsetzen, er kann den verbleibenden Gesellschaftern also die Fortsetzung der Zusammenarbeit in der veränderten personellen Zusammensetzung nicht aufzwingen. Es kann sehr berechtigte Gründe dafür geben, warum diese Perspektive des Austretenden für die Gegner der Kündigungserklärung unattraktiv oder sogar unzumutbar ist, weil sie nach einer Liquidation einen neuen Anfang, u.U. in partiell gleicher Zusammensetzung wagen wollen. Deswegen kommt es auf die konkreten gesellschaftsvertraglichen Abreden an. Soweit der Gesellschaftsvertrag keine gegenteiligen Regelungen enthält, kann kein Gesellschafter, der eine Austrittskündigung eines Mitgesellschafters empfangen hat, gezwungen werden, an der Gesellschaft festzuhalten, vielmehr darf er sich der Kündigung anschließen und kann damit - wenn er der letzte verbliebene Gesellschafter ist - die Beendigung der Sozietät herbeiführen ( $\$ \$ 723$ Abs. 1, $730 \mathrm{ff}$ BGB).

Die Vereinbarung einer Fortsetzungsklausel bzw. eines Übernahmerechts für den letzten verbleibenden Gesellschafter ist nach der Rechtsprechung des Bundesgerichtshofes keine Vereinbarung, die der Ausübung einer solchen Anschlusskündigung entgegensteht. Das gilt selbst bei sog. „Massenkündigungen". ${ }^{2}$

Schließen sich also sämtliche Gesellschafter der von einem Sozius ausgesprochenen Kündigung wirksam an, wird die Gesellschaft aufgelöst und tritt in das Liquidationsstadium ein ( $\$ 730 \mathrm{BGB})$.

\section{Auflösung der Gesellschaft}

Im Falle des Ausscheidens eines Gesellschafters nach $₫ 738$ BGB stellen sich zum Teil dieselben Fragen wie bei der nach der Auflösung der Gesellschaft erforderlichen Auseinandersetzung. Denn auch dann ist hinsichtlich der Ansprüche des ehemaligen Partners zu ermitteln, „was er bei der Auseinandersetzung erhalten würde, wenn die Gesellschaft zur Zeit seines Ausscheidens aufgelöst worden wäre“; es muss also eine fiktive Auseinandersetzungsrechnung erstellt werden. Während diese Aufgabe im Ausscheidensfall den verbleibenden Gesellschaftern zufällt, ist die Situation bei der Auflösung eine andere. Hier sind alle Gesellschafter nicht nur in die Pflicht genommen, für eine ordnungsgemäße Abwicklung zu sorgen, sie haben vor allem auch Rechte, die ihnen nicht genommen werden dürfen.

2 BGH, Beschl. v. 16.11.1998 - II ZR 2/98, DStR 1999, 171 m. Anm. W. GoetTe; BGH, Urt. v. 7.4.2008 - II ZR 181/04, DStR 2008, 1340, 1343. 


\section{a) Gemeinsame Liquidation}

Die Auseinandersetzung der aufgelösten Gesellschaft vollzieht sich gemäß

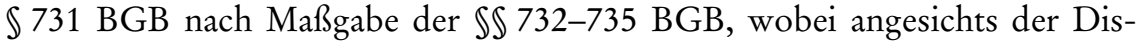
positivität der gesetzlichen Vorschriften etwa vorhandene abweichende Vereinbarungen vorgehen ( $\$ 731 \mathrm{BGB})$. Jeder Gesellschafter ist nach $\$ 730 \mathrm{Abs.} 2$ Satz 2 BGB geborener Liquidator der Gesellschaft, d.h. er hat nicht nur die Pflicht, sondern auch das Recht zur Mitwirkung an der geordneten Abwicklung. Schuldhafte Verstöße gegen diese Pflichten können zu Schadenersatzansprüchen der Mitgesellschafter führen, ${ }^{3}$ die als Einzelposten in die zu erstellende Schlussrechnung einzustellen sind. ${ }^{4}$

Es liegt auf der Hand, dass es nicht nur einen Weg geben kann, wie eine aufgelöste Gesellschaft abgewickelt wird; statt einer zur Zerschlagung der Werte führenden Einzelveräußerung der Gegenstände des Gesellschaftsvermögens kann u.U. die Veräußerung des Ganzen zu einem vorteilhafteren Ergebnis nicht nur für die Gesellschaftsgläubiger, sondern auch für die Gesellschafter selbst führen. Der einzelne Gesellschafter kann ggf. auch ein Interesse daran haben, bestimmte Vermögensgegenstände selbst zu erwerben, weil er sie für einen Neuanfang benötigt.

Gerade bei einer Freiberuflersozietät, deren Partner sich trennen, liegt ein solches Vorgehen nahe, weil typischerweise der wesentliche Wert des Gesellschaftsvermögens immaterieller Art ist, nämlich in der vertrauensvollen Beziehung zu den Mandanten, Klienten oder Patienten besteht, die auch in Zukunft betreuen zu können, die Quelle für das aus der freiberuflichen Tätigkeit zu erzielende Einkommen ist. Vor diesem Hintergrund kann z.B. das einseitige Vorgehen eines Gesellschafters oder einer einzelnen Gruppe, den anderen Partner der aufgelösten Gesellschaft von der Abwicklung auszuschließen und einseitig auf die Mandanten, Klienten oder Patienten einzuwirken, um sie ausschließlich an sich zu binden, schadenersatzpflichtig ${ }^{5}$ machen; das gilt erst recht, wenn dieses pflichtwidrige Verhalten mit der unrichtigen Mitteilung einhergeht, der übergangene Partner sei einverstanden, weil er sich ganz anders orientieren wolle oder weil sich die Gesellschafter auf diese Aufteilung des Gesellschaftsvermögens ausdrücklich geeinigt hätten. Dieser Schadenersatzanspruch muss dann in die später zu erstellende Auseinandersetzungsrechnung als Aktivposten eingesetzt werden, womit die einseitige, die Liquidato-

3 BGH, Urt. v. 9.9.2002 - II ZR 198/00, DStR 2002, 2234; vgl. auch Urt. v. 19.6.1995 - II ZR 255/93, DStR 1995, 1515; C. SCHÄFER, aaO (Fn. 1), \ 730 BGB Rdn. 28.

4 BGH, Urt. v. 27.3.1995 - II ZR 3/94, DStR 1995, 1200; ferner Urt. v. 9.9.2002 - II ZR 198/00, DStR 2002, 2234.

5 BGH, Urt. v. 27.3.1995 - II ZR 3/94, DStR 1995, 1200. 
renpflichten verletzende Aneignung von Gesellschaftsvermögen ausgeglichen wird.

\section{b) Typische Form der Auseinandersetzung einer Freiberuflersozietät}

Die höchstrichterliche Rechtsprechung hat für die Auseinandersetzung einer Freiberuflersozietät in einer jahrelangen Linie von Judikaten immer wieder ausgesprochen, dass die Teilung der Sachwerte und die rechtlich unbeschränkte Möglichkeit, in einen Wettbewerb um die Mandanten der Sozietät zu treten, regelmäßig die "angemessene Art der Auseinandersetzung “ ist. ${ }^{6}$ Dieser Grundsatz, der vor allem auch dann gilt, wenn Ansprüche auf Ausgleich eines good will ausdrücklich ausgeschlossen sind, trägt dem Umstand Rechnung, dass in aller Regel die von den Gesellschaftern aufgebauten Beziehungen zu den Mandanten, Patienten oder Klienten den wesentlichen Wert des Gesellschaftsvermögens ausmachen, dass aber dieser Wert entscheidend auf dem persönlichen Vertrauen zu den einzelnen Mitgliedern der Sozietät beruht, sich folgerichtig als ein „flüchtiges Reh" einer sicheren Übertragung entzieht und sich deswegen auch nicht ohne weiteres kommerzialisieren lässt. Je weniger ausgeprägt dieses auf persönlichem Vertrauen beruhende Verhältnis ist, je mehr es vielmehr um eher standardisierte Dienstleistungen geht, bei denen die Beziehung zum Leistenden nicht im Mittelpunkt steht - ein Beispiel bilden etwa Buchhaltungsaufgaben, wie sie in Steuerberaterpraxen oftmals begegnen -, umso eher wird sich allerdings ein Mandantenstamm - sei es einzeln oder im Ganzen - auf einen Dritten übertragen lassen.

Der besondere Charakter des „Vermögensgegenstandes“ Mandantenstamm hat denn auch zur Folge, dass der Empfänger keineswegs sicher sein kann, die von ihm übernommene Mandantenbeziehung dauerhaft aufrechtzuerhalten und aus ihr in Gestalt von Honorareinnahmen finanzielle Vorteile zu ziehen; deswegen ist die für die Übernahme von Mandanten gezahlte Abfindung dasselbe gilt für den Kaufpreis beim Erwerb eines Mandantenstamms von

6 Vgl. z.B. BGH, Urt. v. 15.1.1990 - II ZR 14/89, ZIP 1990, 1200 f; Urt. v. 6.12.1993 - II ZR 242/92, DStR 1994, 401 m. Anm. W. Goette; Urt. v. 6.3.1995 - II ZR 97/94, DStR 1995, 856 m. Anm. W. Goette; Urt. v. 29.1.1996 - II ZR 286/94, DStR 1996, 1254 m. Anm. W. Goette; Urt. v. 30.9.1996 - II ZR 177/95, DStR 1997, 82 m. Anm. W. Goette; Urt. v. 14.7.1997 - II ZR 238/96, DStR 1997, 1413 m. Anm. W. GoetTE; Beschl. v. 16.11.1998 - II ZR 2/98, DStR 1999, 171 m. Anm. W. GoetTe; Urt. v. 8.5.2000 - II ZR 302/98, DStR 2000, 1273 m. Anm. W. Goette; Urt. v. 8.5.2000 - II ZR 308/98, DStR 2000, 1021 m. Anm. W. GoetTe; (indirekt) Urt. v. 7.3.2005 - II ZR 194/03, DStR 2005, 1018; Urt. v. 7.4.2008 - II ZR 181/04 (und 3/06), DStR 2008, 1340; Beschl. v. 31.5.2010 II ZR 29/09, DStR 2010, 1947; Beschl. v. 14.6.2010 - II ZR 135/09, DStR 2010, 1897; Beschl. v. 18.9.2012 - II ZR 94/10, BeckRS 2012, 22243.

7 BGH, Urt. v. 6.12.1993 - II ZR 242/92, DStR 1994, 401 m. Anm. W. Goette. 
Dritten - nicht mehr als der Preis für die Chance auf Erzielung von Honorar; das Risiko, dass sich diese Chance nicht verwirklicht, liegt allein beim Erwerber.

Wie das dem genannten dictum des II. Zivilsenats hinzugefügte Wort „regelmäßig“ bereits deutlich macht, beschreibt die als „angemessen“ bezeichnete Auseinandersetzungsregelung lediglich einen Grundsatz. Ausnahmen hiervon sind in mehrfacher Hinsicht möglich und waren dementsprechend auch wiederholt Gegenstand höchstrichterlicher Erkenntnisse.

Als Hauptanwendungsfall einer solchen Ausnahme, bei der der unbeschränkte Zugriff auf die Mandanten der Sozietät nicht die angemessene Form der Auseinandersetzung darstellt, ist es anzusehen, wenn im Rahmen der Auseinandersetzung einer aufgelösten Sozietät nicht allen Sozien der Wettbewerb um die Mandanten eröffnet ist. ${ }^{8}$ Die bereits erwähnte Situation, dass einer der Gesellschafter bei der Auseinandersetzung von der Mitwirkung ausgeschlossen ist, der oder die anderen bisherigen Partner vielmehr einseitig auf die Mandanten, Klienten oder Patienten einwirken, die Zusammenarbeit in der Zukunft allein mit ihnen fortzusetzen, ist ein solcher Fall, in dem der Wettbewerb um den Mandantenstamm nicht rechtlich, aber faktisch ausgeschlossen wird. In diesem Fall fehlt es an der Grundvoraussetzung für das die Rechtsprechung in den Normalfällen leitende Prinzip, dass ein unbeschränkter Wettbewerb um das Vertrauen der bisherigen gemeinsamen Mandanten, Klienten oder Patienten eröffnet wird. Der von der treuwidrigen Vorgehensweise des anderen Teils betroffene Gesellschafter muss es nicht hinnehmen, dass sich die Mehrheit der oder alle Mandanten gegen ihn entschieden haben, er also die Chance nicht erhält, eine Mandatsbeziehung mit finanziellem Ertrag aufzubauen. Vielmehr kann er dann verlangen, so gestellt zu werden, als hätte eine ordnungsgemäße also den genannten Grundsatz beachtende - Liquidation stattgefunden, ${ }^{9} \mathrm{~d} . \mathrm{h}$. der entsprechende Schadenersatzanspruch ist in die Auseinandersetzungsrechnung einzustellen. ${ }^{10}$

Es versteht sich von selbst, dass nicht nur bei einem vollständigen Ausschluss eines Liquidators von der Abwicklung der faire, „gleichberechtigte Wettbewerb "11 fehlt, sondern dass es auch vertragswidrig ist, wenn die Chancengleichheit nur teilweise beeinträchtigt wird.

8 Vgl. dazu aus der Rechtsprechung BGH, Beschl. v. 7.5.2007 - II ZR 186/06, DStR 2007, 1491.

9 BGH, Urt. v. 9.9.2002 - II ZR 198/00, DStR 2002, 2234; BGH, Urt. v. 27.3.1995 - II ZR 3/94, DStR 1995, 1200; vgl. auch Urt. v. 19.6.1995 - II ZR 255/93, DStR 1995, 1515 (faktische Liquidation).

10 BGH, Urt. v. 27.3.1995 - II ZR 3/94, DStR 1995, 1200.

11 So ausdrücklich: BGH, Beschl. v. 31.5.2010 - II ZR 29/09, DStR 2010, 1947. 
Eine Verletzung der Liquidatorenpflicht liegt hingegen nicht vor, der betroffene Gesellschafter hat demgemäß auch keine Ansprüche wegen des nicht eröffneten Wettbewerbs um die Mandanten der bisherigen Sozietät, wenn er - trotz entsprechender Aufforderung oder trotz seines Wissens um das Betreiben einer „faktischen Liquidation“ durch die anderen Gesellschafter - untätig bleibt und keine Anstrengungen unternimmt, das Vertrauen dieser Mandanten zu gewinnen und zu ihnen eine auch finanziell erfolgreiche Vertragsbeziehung zu begründen. Das gilt nach der höchstrichterlichen Rechtsprechung vor allem auch dann, wenn der Gesellschafter, der ausgeschieden ist oder der sich in einem Auseinandersetzungsverfahren mit seinen Mitgesellschaftern befindet, von entsprechenden Bemühungen absieht, weil er sie-etwa wegen der starken Bindung der Mandanten an die anderen Sozien - ohnehin nicht für aussichtsreich hält. ${ }^{12}$

Dieses - passive - Verhalten des betreffenden Gesellschafters belegt nämlich nur seine eigene Einschätzung, dass dem Mandantenstamm kein aus der Sicht der Sozietät finanziell messbarer Wert zukommt, sondern das dieser Vermögensgegenstand - ohne besondere Ausgleichspflicht - seinen Mitgesellschaftern zuzuordnen ist ${ }^{13}$

Von dem faktischen - totalen oder partiellen - Ausschluss eines Gesellschafters, am Wettbewerb um die bisherigen Mandanten teilzunehmen, zu unterscheiden sind die Fallgestaltungen, in denen der Wettbewerb rechtlich beschränkt ist. Das ist nicht nur dann anzunehmen, wenn entsprechende Vereinbarungen bestehen, sondern auch - ohne das Vorhandensein entsprechender Abreden einem Gesellschafter eine Abfindung oder die - einer Abfindung der Sache nach gleichstehende - Zahlung von Versorgungsbezügen versprochen worden ist. In allen diesen Fällen ist selbstverständlich - Abfindung wie Versorgungsansprüche sind in diesen Fällen aus dem Ertrag der fortgeführten Verträge mit den Mandanten aufzubringen -, dass der durch diese Abreden begünstigte Gesellschafter nicht zusätzlich um die Mandanten werben darf. ${ }^{14}$ Mitgenommene Mandate - auch wenn dieser Vorgang mangels eines Pflichtverstoßes des

12 BGH, Urt. v. 6.12.1992 - II ZR 242/92, DStR 1994, 401 m. Anm. W. Goette; Beschl. v. 31.5.2010 - II ZR 29/09, DStR 2010, 1947.

13 BGH, Beschl. v. 31.5.2010 - II ZR 29/09, DStR 2010, 1947.

14 Vgl. z. B. BGH, Urt. v. 7.3.1995 - II ZR 194/03, DStR 2005, 1018 (Abfindung zu kürzen bei Missachtung des Wettbewerbsverbots); Urt. v. 29.1.1996 - II ZR 286/94, DStR 1996, 1254 m. Anm. W. Goette (Auswirkung der Kürzung eines überlangen Wettbewerbsverbots); Urt. v. 30.9.1996 - II ZR 177/95, DStR 1997, 82 m. Anm. W. Goette (Versorgung); Urt. v. 14.7.1997 - II ZR 238/96, DStR 1997, 1413 m. Anm. W. GoetTe (Auswirkung nichtigen Wettbewerbsverbots); Urt. v. 8.5.2000 - II ZR 302/98, DStR 2000, 1273 m. Anm. W. Goette (Abfindung in Gestalt eines Gewinnanspruchs für 5 Jahre nach Ausscheiden); Urt. v. 8.5.2000 - II ZR 308/98, DStR 2000, 1021 m. Anm. W. GoetTe (Mandantenschutzvereinbarung); Urt. v. 17.5.2004 - II ZR 261/01, DStR 2004, 1303 (Auswirkung des Praxisverkaufs auf Versorgungsanspruch). 
betreffenden Gesellschafters keine Schadenersatzansprüche auslöst, weil die Mandanten, Klienten oder Patienten aus freien Stücken ihm weiterhin die Treue halten - müssen die zu zahlende Abfindung bzw. den Versorgungsanspruch mindern. ${ }^{15}$ Mit der Chance, Gewinn aus der fortgeführten Mandantenbeziehung zu ziehen, hat der betreffende Gesellschafter alles erhalten, was er nach den genannten Regeln der höchstrichterlichen Rechtsprechung beanspruchen kann, würden Abfindung bzw. Versorgungsanspruch nicht gekürzt, fiele sein Anteil am Liquidationsüberschuss überproportional hoch aus.

Entsprechendes hat bei einer quotalen Teilung des Mandantenstamms zu gelten, so dass eine wegen der freien Entscheidung der Mandanten u.U. eintretende disquotale Verteilung der Mandate Ausgleichsansprüche des Gesellschafters auslösen kann, dessen übernommener Teil des Mandantenstamms hinter seiner Beteiligungsquote zurückbleibt. ${ }^{16}$ Wesentlich ist aber, dass es einen solchen "Spitzenausgleich“ ausschließlich dann geben kann, wenn zwischen den Gesellschaftern eine Aufteilungsvereinbarung auch hinsichtlich des Mandantenstamms besteht. ${ }^{17}$

\section{Beendigung der Mitgliedschaft}

Scheidet dagegen der Partner aus der Gesellschaft aus und wird dieselbe von den verbleibenden Gesellschaftern fortgeführt - die Übernahme des Gesellschaftsvermögen durch den letzten verbleibenden Gesellschafter steht dem gleich - ist eine förmliche Auseinandersetzung, ein Tätigwerden von Liquidatoren nicht veranlasst; der ausscheidende Gesellschafter ist aber abzufinden. Nach $\$ 738$ BGB bestimmt sich - mangels abweichender Abreden - dieser Abfindungsanspruch nach dem, was er bei einer fiktiven Auseinandersetzung hätte fordern können.

15 BGH, Urt. v. 7.3.1995 - II ZR 194/03, DStR 2005, 1018 (Abfindung zu kürzen bei Missachtung des Wettbewerbsverbots); Urt. v. 30.9.1996 - II ZR 177/95, DStR 1997, 82 m. Anm. W. Goette (Versorgung); Urt. v. 14.7.1997 - II ZR 238/96, DStR 1997, 1413 m. Anm. W. Goette (Auswirkung nichtigen Wettbewerbsverbots); Urt. v. 8.5.2000 - II ZR 308/98, DStR 2000, 1021 m. Anm. W. GoetTe (Mandantenschutzvereinbarung) vgl. bildlich gesprochen: „Man kann den Kuchen nicht essen und ihn zugleich behalten wollen“; W. Goette, AnwBl. 2007, $637 \mathrm{ff}, 643$.

16 BGH, Beschl. v. 8.7.1991 - II ZR 285/90, DStR 1991, 1226 (W. GoetTe); Beschl. v. 18.10.1993 - II ZR 41/93, DStR 1993, 1675 (W. GoetTe); Urt. v. 6.3.1995 - II ZR 97/94, DStR 1995, 856 m. Anm. W. Goette; Beschl. v. 16.11.1998 - II ZR 2/98, DStR 1999, 171 m. Anm. W. GoetTe; Beschl. v. 31.5.2010 - II ZR 29/09, DStR 2010, 1947.

17 BGH, Beschl. v. 8.7.1991 - II ZR 285/90, DStR 1991, 1226 (W. GoetTe); Beschl. v. 18.10.1993 - II ZR 41/93, DStR 1993, 1675 (W. GoETTE); Urt. v. 6.3.1995 - II ZR 97/94, DStR 1995, 856 m. Anm. W. GoetTe; Beschl. v. 31.5.2010 - II ZR 29/09, DStR 2010, 1947. 
Damit gilt auch hier: Die Teilung der Sachwerte und die rechtlich unbeschränkte Möglichkeit, in einen Wettbewerb um die Mandanten der Sozietät zu treten, ist regelmäßig die „angemessene Art der Auseinandersetzung “. ${ }^{18}$ Wird auch für den Ausgeschiedenen dieser Wettbewerb ohne Einschränkungen eröffnet, dann liegt es bei ihm, ob er sich auf ihn einlässt und mit welchem Erfolg er ihn besteht. Kann er mehr Gewinnchancen aus auf ihn übergeleiteten Mandaten gewinnen, als seiner Beteiligung an der Gesellschaft entspricht, ist er mangels von der beschriebenen „angemessenen Verteilung“ abweichender Absprachen nicht ausgleichspflichtig; umgekehrt kann er von seinen erfolgreicher um die früheren gemeinsamen Mandanten, Klienten oder Patienten werbenden ehemaligen Partnern keine Kompensation fordern.

Anders ist dies auch hier, wenn rechtliche Beschränkungen des Werbens um Praxis bestehen, also etwa ein Wettbewerbsverbot ausdrücklich vereinbart ist oder naturgemäß als vereinbart gelten muss, weil der ausgeschiedene Partner von den verbleibenden Gesellschaftern eine Abfindung in Geld - u.U. in Gestalt von Versorgungsbezügen - erhält.

In diesem Zusammenhang ist zu berücksichtigen, dass nach der gefestigten Rechtsprechung nicht jede wettbewerbsbeschränkende Abrede unter Freiberuflergesellschaftern der rechtlichen Kontrolle standhält. ${ }^{19}$ Grundsätzlich haben solche Regelungen einen rechtlich anzuerkennenden Sinn, wie man z.B. gerade in dem hier erörterten Zusammenhang mit Abfindungsregeln sehen kann. Sie dürfen aber im Ergebnis nicht dazu führen, dass der betroffene ehemalige Partner mehr als bei einem fairen Interessenausgleich nötig, in seiner beruflichen Entwicklung beschränkt wird. Ein Beispiel dafür ist eine Mandantenschutzklausel - sie ist als solche rechtlich unbedenklich - die so gestaltet ist, dass mitgenommene Mandate mit einem überhöhten Wert auf die Abfindung angerechnet werden müssen. ${ }^{20}$

18 Vgl. z.B. BGH, Urt. v. 15.1.1990 - II ZR 14/89, ZIP 1990, 1200 f; Urt. v. 6.12.1993 - II ZR 242/92, DStR 1994, 401 m. Anm. W. Goette; Urt. v. 6.3.1995 - II ZR 97/94, DStR 1995, 856 m. Anm. W. Goette; Urt. v. 29.1.1996 - II ZR 286/94, DStR 1996, 1254 m. Anm. W. Goette; Urt. v. 30.9.1996 - II ZR 177/95, DStR 1997, 82 m. Anm. W. Goette; Urt. v. 14.7.1997 - II ZR 238/96, DStR 1997, 1413 m. Anm. W. Goette; Beschl. v. 16.11.1998 - II ZR 2/98, DStR 1999, 171 m. Anm. W. GoetTE; Urt. v. 8.5.2000 - II ZR 302/98, DStR 2000, 1273 m. Anm. W. Goette; Urt. v. 8.5.2000 - II ZR 308/98, DStR 2000, 1021 m. Anm. W. GoetTe; (indirekt) Urt. v. 7.3.2005 - II ZR 194/03, DStR 2005, 1018; Urt. v. 7.4.2008 - II ZR 181/04 (und 3/06), DStR 2008, 1340; Beschl. v. 31.5.2010 II ZR 29/09, DStR 2010, 1947; Beschl. v. 14.6.2010 - II ZR 135/09, DStR 2010, 1897; Beschl. v. 18.9.2012 - II ZR 94/10, BeckRS 2012, 22243.

19 S. näher W. Goette, AnwBl. 2007, 637, 642 f.

20 Vgl. BGH, Urt. v. 29.1.1996 - II ZR 286/94, DStR 1996, 1254 m. Anm. W. GoetTe durchschnittlicher Betrag des Gewinns aus den letzten drei Jahren dies jeweiligen Mandats. 
Ähnliches gilt auch für Vereinbarungen, die zu Abfindungsansprüchen führen, die rechtlich nicht hinnehmbar ${ }^{21}$ sind, weil sie z.B. unter dem Buchwert liegen, allein auf Gläubigerbenachteiligung abzielen oder sich so weit vom wahren Wert des Gesellschaftsanteils entfernen oder den Partner - insofern kommt auch eine überlange Dauer der gesellschaftsvertraglichen Bindung in Betracht $^{22}$ - so sehr belasten, dass niemand in der Lage dieses Gesellschafters sich entgegen dem zwingenden $\ 723$ Abs. 3 BGB zu einem Austritt entschließen würde, auch wenn die dafür vorliegenden Gründe mehr als „wichtig“ im Sinne des Gesetzes sind.

\section{Zusammenfassung}

Vorkehrungen, nach der Trennung von den bisherigen Partnern einer Freiberuflersozietät oder nach deren Auflösung nicht unangenehm überrascht zu werden, scheinen - sieht man in dem Bild, welches sich nach der höchstrichterlichen Rechtsprechung bietet, typische Erscheinungen - nicht in ausreichendem Maß getroffen zu werden. Da es sich bei diesen Sozietäten regelmäßig um auf lange Zeit angelegte Zusammenschlüsse von erfahrenen und rechtlich beratenen Berufsträgern handelt, nimmt dies Wunder. ${ }^{23}$ Mehr Sorgfalt - auch in der wohl meist euphorischen Phase der Gesellschaftsgründung auf dieses Vertragswerk zu verwenden und aus dem vorhandenen „Baukasten“ privatautonom die richtigen Werkzeuge zusammen zu stellen, ist dringend anzuraten, nicht nur um nach der Trennung einen guten Neuanfang zu ermöglichen, sondern auch um der Früchte eines längeren erfolgreichen beruflichen Wirkens nicht verlustig zu gehen.

21 S. dazu näher W. GoETTE, StbJb 1996/97, S. $221 \mathrm{ff}$ mit zahlreichen Nachweisen.

22 Vgl. BGH, Urt. v. 18.9.2006 - II ZR 137/04, DStR 2007, 34 m. Anm. W. Goette überlange Dauer flankiert durch eine höchst belastende Versorgungsregelung zugunsten des Altsozius.

23 S. schon W. Goette, AnwBl. 2007, 637. 